\title{
PROPUESTA DE SECUENCIA DIDÁCTICA UTILIZANDO EL ÁBACO NEPOHUALTZINTZIN PARA LOS CÁLCULOS ARITMÉTICOS
}

\author{
Didactic Sequence Proposal Using the Nepohualtzintzin \\ Abacus for Arithmetc Calculations
}

María del Socorro Rivera Casales ${ }^{1}$

soco_rcms@yahoo.ocm.mx

\section{Ricardo Jacob Mendoza-Rivera²}

ricardo.mendoza@ciencias.unam.mx

Andrea Cárdenas Mendoza ${ }^{3}$

andreacm@acua-edu.mx

${ }^{1}$ Universidad Nacional Autónoma de México. Colegio de Estudios de Posgrado de la Ciudad de México

${ }^{2}$ Universidad Nacional Autónoma de México ${ }^{3}$ Colegio de Estudios de Posgrado de la Ciudad de México

Recibido: 25/10/2019

Revisado: 3/02/2020

Aprobado: 12/10/2020

Preprint: 10/03/2021

Publicado: 15/07/2021

\section{Resumen}

La presente investigación educativa tiene por objetivo diseñar una propuesta de secuencia didáctica identificando los elementos teóricos, metodológicos y herramientas para el uso del ábaco Nepohualtzintzin en las operaciones aritméticas, cuyo fin es desarrollar habilidades con un aprendizaje significativo. El enfoque es cualitativo, usando la observación y cuestionarios. La investigación se realizó en tres etapas: documental, indagación y análisis. Los resultados obtenidos en el estudio de caso en su etapa inicial (cuestionario diagnóstico de conocimientos aritméticos) señalan que el $92 \%$ de los estudiantes tiene un rezago en la enseńanza del aprendizaje memorístico por descubrimiento autónomo y no llegan a un aprendizaje-significativo (aprendizaje a largo plazo). La revisión y el análisis de la propuesta institucional que ofrece la UNAM para la enseñanza de Matemáticas en la Escuela Nacional Preparatoria (ENP) proporcionaron evidencias de la saturación de contenidos y no de la comprensión que se otorga en el nivel medio-superior a la enseñanza de conceptos y al razonamiento matemático (Rivera, 2012a), de modo que solo se logra en la enseńanza un aprendizaje memorístico-receptivo.

Palabras clave: ábaco, aprendizaje significativo, habilidades matemáticas, Nepohualtzintzin, secuencia didáctica.

\section{Abstract}

The purpose of this educational research is to design a proposal of didactic sequence identifying the theoretical, methodological elements and tools for the use of the Nepohualtzintzin abacus in arithmetic operations, in order to develop skills with significant learning. The approach is qualitative and its technique is observation and questionnaires. The research was conducted out in three stages: documentary, inquiry and analysis. The results obtained in the case study in its initial stage (diagnostic questionnaire of arithmetic knowledge), indicates that $92 \%$ of the students lag behind in teaching rote learning by discovery-autonomous and do not reach significant-learning (long-term learning). The review and analysis of the institutional proposal offered by the UNAM for the teaching of Mathematics at the National Preparatory School (ENP), provided evidence of content saturation and not of the understanding that is given at the upper-middle level of teaching of concepts and mathematical reasoning (Rivera, 2012a), so that only rote-receptive learning is achieved in teaching.

Keywords: abacus, didactic sequence, nepohualtzintzin, mathematics skills, significant learning. 


\section{Introducción}

La presente investigación tiene por objetivo diseñar una propuesta de secuencia didáctica identificando los elementos teóricos, metodológicos y las herramientas para utilizar el ábaco Nepohualtzintzin como material didáctico, en relación con los cálculos aritméticos en un estudio de caso de alumnos en la Escuela Nacional Preparatoria (ENP).

La hipótesis de la presente investigación supone que el diseño de una secuencia didáctica con un aprendizaje significativo constructivista, realista, lúdico y cooperativo favorece la disminución del rezago existente en operaciones aritméticas en los estudiantes de la ENP.

La originalidad de este trabajo reside en destacar una herramienta didáctica mesoamericana, el ábaco Nepohualtzintzin. El problema de investigación está centralmente referido a la enseńanza-aprendizaje, es decir, el estudio, además de analizar problemas aritméticos a partir de la realidad actual, permite deliberadamente promover la "reconstrucción mental de dicha realidad", para lo cual se diseña una secuencia didáctica que se adecua a los contenidos matemáticos propuestos en los planes y programas de estudio de la ENP. La realidad es que se debe enseñar de manera continua, con la finalidad de "educar, aprender y adiestrarse para la vida” (ENP, 1996).

La pregunta central de la investigación es: ¿Cuáles son los elementos, teóricos, metodológicos y las herramientas necesarias para elaborar una propuesta de secuencia didáctica utilizando el ábaco Nepohualtzintzin como material didáctico para los cálculos aritméticos (etnomatemática)?

La propuesta de la secuencia didáctica utilizando el ábaco Nepohualtzintzin en la ENP, en la que el alumno realizará actividades para ir desarrollando un aprendizaje significativo, reforzará los conocimientos de forma visual y táctil, tomando en cuenta las aportaciones históricas prehispánicas y conceptos esenciales. El profesor fomentará una clase dinámica utilizando el ábaco.

El presente proyecto de investigación cuenta con el acceso a la Escuela Nacional Preparatoria Plantel No.
2 "Erasmo Castellanos Quinto", de la Universidad Nacional Autónoma de México, con una muestra de 37 alumnos del grupo 417, durante el ciclo escolar 2019-2020.

\section{Revisión de la literatura}

El desempeño de los alumnos de Educación Básica en México en el ámbito de la Matemática ha sido deficiente en los últimos años. Los resultados de la prueba PISA (Programme for International Student Assessment) indican OCDE (2016) que el desempeño de México se ubicó por debajo del promedio en Ciencias (416 puntos), Lectura (423 puntos) y Matemáticas (408 puntos). En estas tres áreas, menos del $1 \%$ de los estudiantes mexicanos logran alcanzar excelentes niveles de competencia (niveles 5 y 6 ).

En México, el gasto en Educación Básica implica el 17\% del presupuesto total. En 2015 destinó un acumulado de 29,015 USD por estudiante, menos de un tercio del promedio de gasto de la OCDE, que ascendió a 90,561 USD, según el estudio de la OCDE Panorama Educativo 2018. Esta cifra es significativamente mayor que la de otros países latinoamericanos en desarrollo, sin embargo, este flujo no se ve potencialmente invertido en herramientas que sustenten el desarrollo del alumno.

Diversos estudios enfocan la educación como una inversión para impulsar el desarrollo económico y social de un país, pero esta inversión debe ser correctamente gastada e invertida en todos los sectores de la enseñanza. López et al. (2005) muestran que el desarrollo sostenible debe significar una inversión constante y debe involucrar tanto al sector público como al privado.

Herramientas como el ábaco Nepohualtzintzin permiten impulsar el aprendizaje significativo y fomentar la identidad del estudiante mexicano. Además, sabemos que mundialmente el empleo de un ábaco en la Educación Básica ha mostrado un desarrollo de la lógica, la lingüística y el fomento de la aritmética.

Una problemática en la enseñanza de las Matemáticas en cualquier nivel es la deficiencia en la 
enseńanza-aprendizaje de los cálculos aritméticos. Algunas de las causas observadas en el estudio dirigido a los estudiantes de la Escuela Nacional Preparatoria son:

1. Desconocimiento de la etnomatemática.

2. Desconocimiento del ábaco Nepohualtzintzin.

3. Falta de atención a la enseñanza-aprendizaje de la historia cultural prehispánica y de la identidad mexicana.

4. Falta de dominio en los estudiantes de bachillerato universitario al realizar cálculos aritméticos.

5. Falta de interés de los alumnos al resolver operaciones aritméticas.

Estos problemas crean un rezago en la enseñanza de las Matemáticas. De allí que los docentes de niveles posteriores siempre tienen una queja sobre la falta de dominio de los conceptos básicos. Esta investigación busca apoyar esta labor, pues "El papel del docente en el contexto actual del mundo globalizado es proporcionar, mediante estrategias planificadas, actividades y recursos, una guía constante a los estudiantes, dando la libertad para que a partir de los conocimientos previos con los que cuentan, las actividades y los recursos que se les proporcionan, los estudiantes construyan y formen su educación" (Cárdenas, 2016, p. 29).

\subsection{Rezago educativo}

El rezago educativo es un aplazamiento de conceptos básicos que cada estudiante debe tener para cursar el siguiente nivel escolarizado. Se le atribuye a un conjunto de factores externos a los sistemas escolares, que se deriva de una problemática existente en la sociedad de cada cultura y país. "Este término se utiliza en el primer estudio de largo alcance que fue realizado con el propósito de identificar la problemática que tienen los diferentes sistemas educacionales en la determinación del aprovechamiento académico de los estudiantes. Ese estudio se conoce con el nombre de Reporte Coleman" (Coleman et al., 1966). El autor dedujo de sus análisis que las escuelas desempeñan un papel meramente pasivo en la determinación del rendimiento de sus alumnos. De esta interpretación se desprende que el sistema escolar reproduce las desigualdades sociales, sin poder combatirlas.

\subsection{Secuencia didáctica}

Las secuencias didácticas construyen una organización en las actividades del aprendizaje que se realizarán con los alumnos y para los alumnos, con la finalidad de crear situaciones que les permitan desarrollar un aprendizaje significativo. Acompañan la perspectiva didáctica del docente mediante una guía que le permitirá la construcción de secuencias didácticas.

\subsubsection{Estructura de una secuencia didáctica}

La estructura de una secuencia didáctica establece cuatro actividades de aprendizaje que tengan un orden interno adecuado entre ellas. El docente comienza recuperando las nociones previas que tienen los estudiantes sobre un tema estudiado, mediado por la propuesta institucional, estableciendo una vinculación a situaciones problemáticas y a contextos reales para que el alumno les encuentre un significado (Brousseau, 2007). La estructura de la secuencia integra:

a) Actividades de apertura.

b) Actividades de desarrollo.

c) Actividades de cierre.

d) Línea de evaluación para el aprendizaje.

El papel de las actividades de la secuencia didáctica y la forma de realizar cada una de ellas es lo que se denomina "línea de secuencias de aprendizaje" (Anijovich, 2010).

\subsection{Aprendizajes}

David Ausubel (1976) afirma que, dentro del aprendizaje significativo, el estudiante debe relacionar de manera primordial la nueva información con sus conocimientos y experiencias previas. Se requiere de mucha disposición del estudiante para aprender significativamente, y la mediación del 
docente es fundamental en esa dirección. Por otro lado, es muy importante la forma en que se plantean los materiales de estudio y las experiencias educativas. Si se llega al aprendizaje significativo, se trasforma la repetición memorística de resolución de problemas y se logra construir un significado en cada tema abordado por el docente, dando sentido a lo aprendido, así como entendiendo su ámbito de aplicación y relevancia en situaciones reales académicas y cotidianas.

Hasta ahora se ha insistido en la continuidad que existe entre el modo y la forma en que se adquieren los conocimientos en relación con las posibles situaciones de aprendizaje escolar. En las categorías se ejemplificarán las dimensiones de los aprendizajes intelectuales, memorísticos y significativos (Novak \& Gowin, 1988; García-Madruga, 1991).

Tipos de aprendizaje (Díaz-Barriga, 2010):

a) Significativo:

- Receptivo. Relaciones entre conceptos.

- Por descubrimiento guiado. Instrucción bien diseñada.

- Por descubrimiento autónomo. Investigación científica.

b) Memorístico:

- Receptivo. Algoritmos matemáticos.

- Por descubrimiento guiado. Aplicación de fórmulas.

- Por descubrimiento autónomo. Solución de acertijos.

\section{4. Ábaco Nepohualtzintzin}

El término ábaco procede del griego abax o abakon, que significa 'superficie plana' o 'tabla cubierta de polvo'. En un principio utilizaban fichas o pequeñas piedras (calculi) sobre una mesa o una bandeja plana en la cual separaban zonas diferentes, correspondientes a órdenes de unidades, utilizando líneas que marcaban con polvo (López, 2008, p. 153).

El diccionario de la Real Academia Española define ábaco como "Bastidor con cuerdas o alambres paralelos y en cada uno de ellos bolas móviles, usado para operaciones elementales de aritmética”.

Esta herramienta didáctica mesoamericana es empleada para calcular o contar. Se denominan ábacos mesoamericanos y poseen características propias de acuerdo con su uso en cada región específica.

La etnomatemática de la cultura maya ocupó el territorio del sur de México, a través de Guatemala, Honduras y parte de El Salvador, extendiéndose al sur hasta Panamá y al norte hasta la costa mexicana en Pánuco. La cultura maya tuvo un gran desarrollo, que se observa en los sistemas de numeración y en una matemática astronómica avanzada, con varios calendarios de gran exactitud que implicaban extensas cuentas y cálculos, la asombrosa precisión de la construcción de sus pirámides, palacios y templos, muchos de los cuales se encuentran protegidos hoy en día (López, 2008).

El sistema numérico maya era vigesimal (base igual a 20), posicional, y empleaba un símbolo para representar la ausencia de valor en una determinada posición. Su mayor relevancia es haber usado el cero en forma de concha y números muy grandes, asociando un dios a cada número — del 0 al 19-. Dichos números se representaban con puntos y rayas, tal como se muestra en la Figura 1.

\section{Figura 1. Representación del sistema de numera- ción maya}

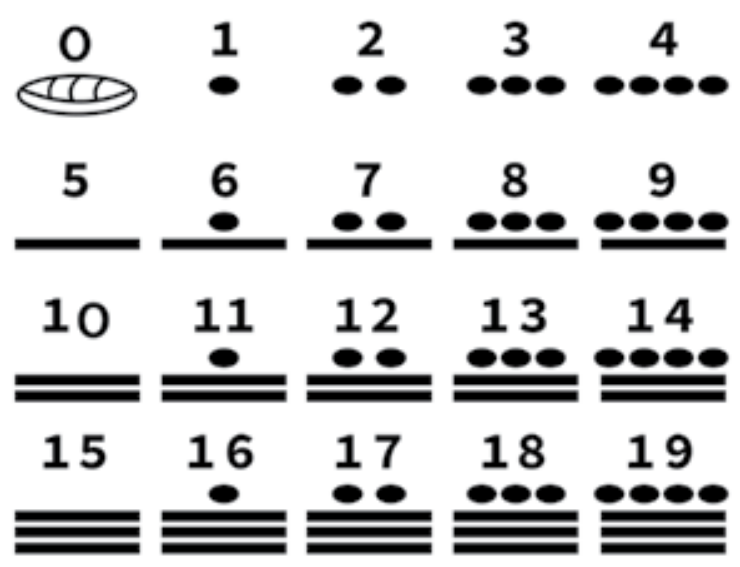

Nota: Elaboración propia. 


\subsubsection{Descripción del ábaco Nepohualtzintzin, con adaptación al sistema decimal}

El ábaco es una herramienta didáctica idónea para cálculos aritméticos en Educación Básica. Es una de las herramientas didácticas más antiguas, que permite manipular y visualizar de forma clara los números por conteo, así como entender la estructura de las unidades, decenas y centenas. Sumar, restar, multiplicar, dividir, etc., son algunas de las principales operaciones que se pueden efectuar con este instrumento (Vázquez, 2010).
La estructura del ábaco es la siguiente: tiene una sección de cuatro cuentecitas, una barra central y otra sección de tres cuentas. La sección que tiene cuatro cuentas equivale al modelo de los cuatro dedos de la mano, menos uno, el pulgar, ya que la mano completa está simbolizada en las cuentas de la sección de tres cuentas, donde cada una tiene el valor de una mano, es decir, cinco. El Nepohualtzintzin cuenta con 13 columnas, líneas o ejes verticales, cada una representa las articulaciones mayores del cuerpo: dos tobillos, dos rodillas, dos ingle, dos muñecas, dos codos, dos hombros y el cuello (Soto, 2014); se muestra en la Figura 2.

\section{Figura 2. Descripción del ábaco Nepohualtzintzin}

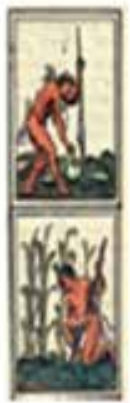

- 1 cuello

- 2 hombros

- 2 codos

- 2 coxofemorales

- 2 muñecas

- 2 rodillas

- 2 tobillos

- 13 articulaciones

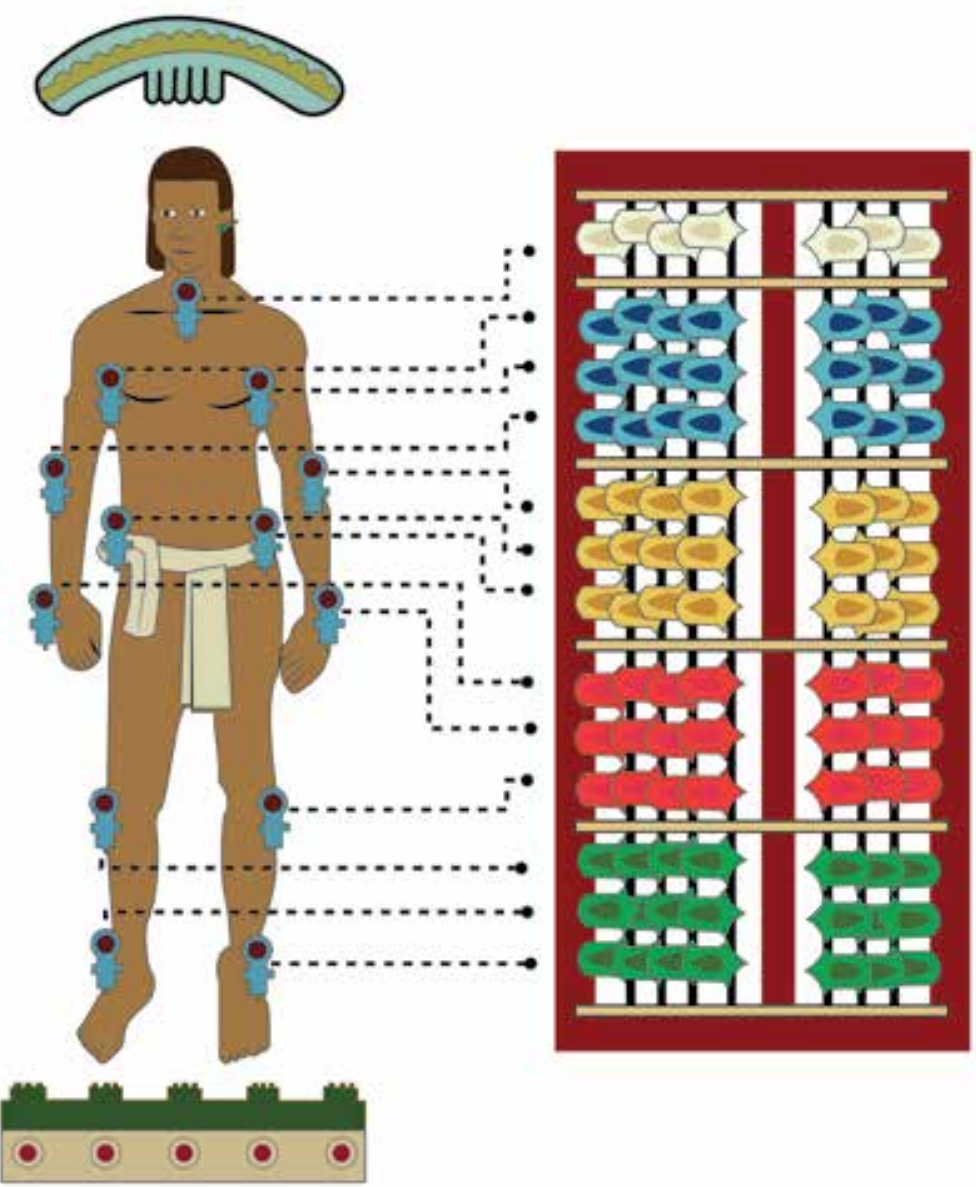




\subsubsection{Etnomatemática}

Durante la conquista de España, los pueblos mesoamericanos mexicanos tenían desarrollados sus propios conocimientos matemáticos, muy propios de cada cultura, influidos por sus propias creencias, tradiciones, usos y costumbres.

La definición de etnomatemática proviene del griego ethnos y mathema, conocer, explicar, entender, y ties, vinculado al arte o a la técnica. El termino etnomatemática es un concepto más amplio de la matemática, que no solo es contar ni hacer únicamente operaciones aritméticas o medir, sino que también implica clasificar, ordenar, inferir, modelar, ver la naturaleza y el cosmos (D’Ambrosio, 2008).

En la etnomatemática no solo se modelan los conocimientos matemáticos vistos y analizados por los grupos étnicos, también se observan otros aspectos: antropológico, histórico, geográfico y filosófico de cada grupo, los cuales inciden en este conocimiento matemático. D'Ambrosio resume la etnomatemática como "la matemática practicada por grupos culturales" (Jaramillo et al., 2006, p. 2).

\section{Método}

\subsection{Enfoque del estudio}

- La investigación posee un enfoque cualitativo (Eisner, 1998).

- Método de investigación-acción: es un estudio de la situación actual con el fin de mejorar la calidad de la acción innovando mediante el ábaco Nepohualtzintzin.

- Técnica: observación (secuencia didáctica) y cuestionarios.

Las cualidades de esta investigación son:

1) Trabajar la investigación en el aula real, con determinados estudiantes y maestros, en la institución definida en el momento y espacio determinados.
2) La intervención y participación de la investigadora en la investigación, como guía y observadora.

3) El enfoque interpretativo de la investigación cualitativa, usando una técnica de observación y aplicación de los cuestionarios al inicio del curso y al término de la secuencia didáctica.

4) Los análisis tratan de justificar la problemática planteada. En un segundo sentido, se observa lo fundamental identificado en la situación estudiada, se refiere al uso del lenguaje oral y escrito, la observación. La investigación intenta comprender lo que las personas realizan (Ojeda, 2006).

\subsection{Espacios para la investigación propuesta}

Los espacios para el desarrollo de esta investigación parten primordialmente de la propuesta institucional.

Este estudio no obedeció a un patrón dado, sino que, de acuerdo con el desempeño de cada uno de los participantes, se podían realizar cambios que se consideraran pertinentes; por ejemplo, en caso de que el docente del grupo requiriese la intervención de la investigadora para la aplicación del tema a los estudiantes, la investigadora podía explicar ese tema.

\subsubsection{Primera etapa, documental: propuesta ins- titucional (elaboración de la secuencia didáctica, los cuestionarios y la guía de observación)}

Se dirigió una investigación documental al programa de estudio de Matemáticas IV en el bachillerato universitario (Programa de estudios de ENP, 1996, clave 1400), para la elaboración de la secuencia didáctica y los cuestionarios exploratorios antes y después del curso.

Actividades de apertura. Presentar la organización de las sesiones de enseñanza mediante la secuencia didáctica, para dar una solución al rezago existente en operaciones aritméticas, mediada por la propuesta institucional, ya que la obligación del docente es guiarse por las propuestas institucionales. 


\subsubsection{Segunda etapa, indagación: caracterización indirecta de las sesiones de enseńanza en el aula}

Se realizó una gestión para el acceso al aula. Se acordaron con la docente titular del grupo participante las condiciones en las que se desarrollaría la enseñanza. Luego, antes y después de su práctica, en el aula se procedió a la aplicación de dos cuestionarios.

En el aula se desarrolló la práctica de la secuencia didáctica, conducida por la docente titular del grupo. Los análisis de las sesiones de enseñanza de aritmética en el aula proporcionaron la materia prima para esta etapa de la investigación.

Actividades de desarrollo. Aplicar el cuestionario diagnóstico de conocimientos aritméticos antes del curso y analizarlo para diseñar las 25 sesiones de enseñanza (Rivera, 2012b), mediadas por la propuesta institucional, la etnomatemática y el ábaco Nepohualtzintzin (Díaz-Barriga, 2004).

\subsubsection{Tercera etapa, de análisis (guía de observa- ción)}

En esta etapa mediada por la guía de observación categórica durante el curso participaron 37 alumnos del bachillerato universitario.

Cada una de las etapas se desarrolló en tiempos diferentes, como se describe a continuación. La primera etapa, parte de la segunda y de la tercera se realizaron durante el ciclo escolar 2019-2020. Más específicamente, en la primera etapa, con duración de tres meses, se analizó la propuesta institucional para la realización de la secuencia didáctica y los cuestionarios; en la segunda etapa, con duración de diez meses, se aplicaron y analizaron los dos cuestionarios administrados, uno antes y otro después de la secuencia didáctica. La guía de observación en el aula se realizó en sesiones de 50 minutos (en los horarios asignados al grupo). La tercera etapa requiere de 10 meses para realizar los análisis.

Actividades de cierre. Aplicar el cuestionario diagnóstico de conocimiento después de las sesiones de enseñanza mediadas por la secuencia didáctica.
Línea de evaluación para el aprendizaje. Los análisis de los cuestionarios diagnósticos mediados por la guía de observación categórica, para dar una alternativa de solución al rezago existente en las operaciones aritméticas.

\section{Resultados}

Se presenta en este apartado el comienzo de la investigación, con la aplicación y el análisis del cuestionario diagnóstico al inicio del curso en el estudio de caso de los 37 estudiantes, para la realización de las sesiones de enseñanza mediadas por la secuencia didáctica.

El análisis del cuestionario, antes de las sesiones de enseńanza, da como resultado:

1) Al preguntar si conocían qué estudia la etnomatemática, los 37 alumnos respondieron que no sabían qué estudiaba (ni que existía). Esta pregunta es importante para el diagnóstico y rescate de las culturas mesoamericanas.

2) Al preguntar si conocían el ábaco Nepohualtzintzin, todos respondieron que no lo conocían. Para los investigadores, es relevante conocer y destacar la grandeza de la cultura prehispánica y rescatarla, a fin de mejorar la enseñanza-aprendizaje.

3) Existe una falta de atención a la enseñanza-aprendizaje de la historia cultural prehispánica, pues en los programas de historia de la ENP no dan historia mesoamericana (prehispánica), solo historia después de la conquista en la materia Historia Universal (ENP, 1996, clave 1403).

4) Falta de dominio en los estudiantes de bachillerato universitario al realizar cálculos aritméticos. En la Tabla 3 daremos respuesta a la problemática planteada, ofreciendo los resultados del cuestionario diagnóstico al inicio del ciclo escolar.

5) Otro problema es la falta de interés de los alumnos en el compromiso de resolver operaciones aritméticas. 
Los resultados del análisis de aprendizaje del estudio son los siguientes: tienen un dominio del 68\% en aprendizaje memorístico-receptivo, un $84 \%$ en aprendizaje por descubrimiento guiado y un $28 \%$ en aprendizaje por descubrimiento autónomo. El 92\% de los alumnos no logra alcanzar los niveles de excelencia por tener un aprendizaje memorístico-receptivo, mientras que el $8 \%$ tiene un aprendizaje memorístico por descubrimiento autónomo. Todavía no se llega a los niveles de excelencia que evalúa la prueba PISA: el tiempo máximo para su solución fue de 53 minutos, el mínimo es de 25 minutos y el promedio de tiempo para resolver el cuestionario fue de 39 minutos. Esto da como resultado un aprendizaje a corto plazo.
Se observa en el análisis de la Tabla 2 el desinterés por resolver los reactivos $(1,2,3,4,5,6,7,8,9,10$, 11,13 y 14 ) del $23 \%$ de los 37 alumnos. El desinterés lo podemos interpretar así: no leer bien lo solicitado, falta de dominio de los temas asignados, estar enfermo, flojera para resolver los ejercicios, no haber comido bien, deseo de terminar rápido. El tiempo que tardaron estos alumnos en resolver el cuestionario fue de 27 minutos.

Esta problemática crea un rezago en la enseñanza de las Matemáticas en los cálculos de operaciones aritméticas; los docentes de niveles posteriores siempre tienen una queja por la falta de dominio de los conceptos básicos.

Tabla 1. Resultados del cuestionario diagnóstico antes del curso, en el estudio de caso de 37 alumnos del bachillerato universitario

\begin{tabular}{|c|c|c|c|c|}
\hline & Reactivo & $\begin{array}{l}\text { Respuesta } \\
\text { del reactivo }\end{array}$ & $\begin{array}{l}\text { Total de } \\
\text { respuestas } \\
\text { correctas al } \\
\text { reactivo }\end{array}$ & Dominio de aprendizaje \\
\hline 1. & $5^{2}$ & 25 & 30 & $\begin{array}{l}\text { Aprendizaje memorístico- } \\
\text { receptivo }\end{array}$ \\
\hline 2. & $957 \times 86$ & 82302 & 35 & $\begin{array}{l}\text { Aprendizaje memorístico- } \\
\text { receptivo }\end{array}$ \\
\hline 3. & $84.5+0343$ & 84.843 & 32 & $\begin{array}{l}\text { Aprendizaje memorístico- } \\
\text { receptivo }\end{array}$ \\
\hline 4. & $530 \div 7$ & 75.714 & 29 & $\begin{array}{l}\text { Aprendizaje memorístico- } \\
\text { receptivo }\end{array}$ \\
\hline 5. & $38.9-0.845$ & 38.055 & 25 & $\begin{array}{l}\text { Aprendizaje memorístico- } \\
\text { receptivo }\end{array}$ \\
\hline 6. & $(3-8)+[-(-2)]$ & 2 & 26 & $\begin{array}{l}\text { Aprendizaje memorístico- } \\
\text { receptivo }\end{array}$ \\
\hline 7. & $5-[6-2-(1-8)-3+6]+5$ & -4 & 20 & $\begin{array}{l}\text { Aprendizaje memorístico- } \\
\text { receptivo }\end{array}$ \\
\hline 8. & {$\left[(17-15)^{3}+(7-12)^{2}\right] \div[(6-7) \cdot(12-23)]$} & 3 & 13 & $\begin{array}{l}\text { Aprendizaje memorístico- } \\
\text { receptivo }\end{array}$ \\
\hline 9. & $(7-2+4)-(2-5)$ & 12 & 21 & $\begin{array}{l}\text { Aprendizaje memorístico- } \\
\text { receptivo }\end{array}$ \\
\hline 10. & $6+\{4-[17-(4 \cdot 4)]+3\}-5$ & 7 & 21 & $\begin{array}{l}\text { Aprendizaje memorístico- } \\
\text { receptivo }\end{array}$ \\
\hline 11. & $\begin{array}{l}\text { Teresa ve en el pizarrón la siguiente fracción: } \\
10.17 \text { ¿Cuál de las siguientes opciones muestra } \\
\text { correctamente en escritura decimal esa fracción? } \\
\text { A) Un entero, siete décimos. } \\
\text { B) Diez y siete milésimos. } \\
\text { C) Diez y siete centésimos. } \\
\text { D) Un entero, siete centésimos. }\end{array}$ & $\mathrm{C}$ & 25 & $\begin{array}{l}\text { Aprendizaje memorístico por } \\
\text { descubrimiento guiado }\end{array}$ \\
\hline
\end{tabular}


Reactivo

\section{Respuesta Total de \\ del reactivo respuestas \\ correctas al \\ reactivo}

D
Dominio de aprendizaje

37 Aprendizaje memorístico por descubrimiento guiado cada país cuenta con 4 canoas y cada canoa cuenta con 4 participantes. ¿Cuántos participantes hay en total dentro de la competencia?

A) 12 B) 20 C) 32 D) 64

13 El área de un muro rectangular es de $3,230 \mathrm{~m}^{2}$, si la $10.352 \mathrm{~m}^{2}$

16 Aprendizaje memorístico por base mide $312 \mathrm{~m}$, ¿cuántos metros mide la altura del muro?

A) 7,113 B) 3,128 C) 3,132 D) 9,571

(No hay respuesta correcta, el alumno debe descubrirla)

14. Juan va a colocar losetas de $0.30 \mathrm{~m} \times 0.30 \mathrm{~m}$ en el piso de una de las habitaciones de su casa que mide 167 losetas

5 Aprendizaje memorístico por $15 \mathrm{~m}^{2}$ : ¿cuál es el número aproximado de losetas que empleará?

A) 14.97 B) 15.48 C) 43 D) 86

(No hay respuesta correcta, el alumno debe descubrirla)

Total de respuestas $14 \times 37=518$

Nota: En México la notación para representar centésimos es con punto “.” y miles con coma”,”.

Tabla 2. Análisis de la falta de interés en la resolución de ejercicios en el estudio de caso de alumnos del bachillerato universitario (13-14 años)

\begin{tabular}{|c|c|c|}
\hline & Respuesta de la aplicación del reactivo & $\begin{array}{l}\text { Análisis de falta de interés en la resolución de } \\
\text { ejercicios }\end{array}$ \\
\hline 1. & $5^{2}=10$ & Se le olvida multiplicar $5 \times 5$ y hace $5 \times 2$ \\
\hline 2. & $957 \times 86=82,301$ & No realiza operaciones \\
\hline 3. & $84.5+0.343=84.348$ & No realiza correctamente sus operaciones \\
\hline 4. & $530 \div 7=740$ & Realiza y borra las operaciones \\
\hline 5. & $38.9-0.845=39.745$ & Falta de atención a la operación \\
\hline 6. & $(3-8)+[5-(-2)]=-35$ & No realiza correctamente sus operaciones \\
\hline 7. & $5-[6-2-(1-8)-3+6]+5=4$ & No realiza correctamente sus operaciones \\
\hline 8. & {$\left[(17-15)^{3}+(7-12)^{2}\right] \div[(6-7) \cdot(12-23)]=3$} & No realiza correctamente sus operaciones \\
\hline 9. & $(7-2+4)-(2-4)=2$ & No realiza correctamente sus operaciones \\
\hline 10. & $6+\{4-(17-(4 \cdot 4)]+3\}-5=1$ & No realiza correctamente sus operaciones \\
\hline 11. & $\begin{array}{l}\text { Teresa ve en el pizarrón la siguiente fracción: } 10.17 \text { ¿Cuál de las } \\
\text { siguientes opciones muestra correctamente en escritura decimal } \\
\text { esa fracción? } \\
\text { Respuesta: } \\
\quad \text { A) Un entero, siete décimos. }\end{array}$ & No lee bien la pregunta planteada en el problema \\
\hline 12. & $\begin{array}{l}\text { En una competencia de canotaje compiten } 4 \text { países, cada país } \\
\text { cuenta con } 4 \text { canoas y cada canoa cuenta con } 4 \text { participantes. } \\
\text { ¿Cuántos participantes hay en total dentro de la competencia? } \\
\text { Respuesta: } \\
\text { D) } 64\end{array}$ & $\begin{array}{l}\text { Resuelve correctamente el ejercicio sin necesidad } \\
\text { de operaciones }\end{array}$ \\
\hline
\end{tabular}




\section{Respuesta de la aplicación del reactivo}

Análisis de falta de interés en la resolución de ejercicios

13 El área de un muro rectangular es de $3,230 \mathrm{~m}^{2}$, si la base mide $312 \mathrm{~m}$, ¿cuántos metros mide la altura del muro?

(No hay respuesta correcta, el alumno debe descubrirla) Respuesta:

B) 3,128

Justificación del alumno:

$$
\begin{gathered}
A=3230 \\
\text { base }=312
\end{gathered}
$$

Plantea correctamente la operación, pero no la resuelve:

$$
\frac{312}{230}=103 .+1100
$$

14. Juan va a colocar losetas de $0.30 \mathrm{~m} \times 0.30 \mathrm{~m}$ en el piso de una de las habitaciones de su casa que mide $15 \mathrm{~m}^{2}$ : ¿cuál es el número aproximado de losetas que empleará?

A) 14.97 B) 15.48 C) 43 D) 86

(No hay respuesta correcta, el alumno debe descubrirla)

Respuesta:

D) 86

Justificación del alumno:

Primero interpreta que el área de la loseta es:

Plantea varios escenarios para encontrar la respuesta

$15 \times 9=135$

$312 \times 3=936 m^{2}$
No lee correctamente el ejercicio, descuida las operaciones con punto decimal y no interpreta correctamente la respuesta
Se observa en promedio que el 36\% (14) de los estudiantes tiene un rezago en operaciones aritméticas con un aprendizaje memorístico-receptivo antes del curso. Las sesiones de enseñanza se deben realizar fortaleciendo los temas en los que tuvieron más errores (reactivos 5, 6, 7, 8, 9, 10, 11, 13 y 14), y un $92 \%$ tiene un rezago en la enseñanza del aprendizaje memorístico por descubrimiento autónomo (llegar a un aprendizaje a largo plazo), como lo muestra el análisis de la Tabla 3.

\section{Discusión y conclusiones}

En promedio, el 36\% de los estudiantes tiene un rezago en operaciones aritméticas con un aprendizaje memorístico-receptivo antes del curso. Por ello es necesario dar una alternativa de solución al problema del rezago existente en el estudio de caso. Presentamos en la Tabla 4 la secuencia didáctica; la estructura muestra los temas del curso relacionados con los temas que se deben reforzar (ver el cuestionario de la Tabla 2). Reactivos: 1, 2, 3, 4, 5, 6, 7, 8, 9, 10, 11 , 13 y 14 . 
Tabla 3. Análisis del rezago existente en el estudio de caso de los 37 alumnos del bachillerato universitario (13-14 ańos)

\begin{tabular}{|c|c|c|}
\hline & Reactivo & Análisis de rezago en operaciones aritméticas \\
\hline 1. & $5^{2}$ & $\begin{array}{l}\text { Existe un } 19 \% \text { de rezago en el tema de potenciación y } \\
\text { radicación. }\end{array}$ \\
\hline 2. & $957 \times 86$ & $\begin{array}{l}\text { Existe un } 5 \% \text { de rezago en el tema de números enteros y } \\
\text { sus operaciones, multiplicación. }\end{array}$ \\
\hline 3. & $84.5+0.343$ & $\begin{array}{l}\text { Existe un } 14 \% \text { de rezago en el tema de números enteros y } \\
\text { sus operaciones, suma con números decimales. }\end{array}$ \\
\hline 4. & $530 \div 7$ & $\begin{array}{l}\text { Existe un } 22 \% \text { de rezago en el tema de números enteros y } \\
\text { sus operaciones, división. }\end{array}$ \\
\hline 5. & $38.9-0.845$ & $\begin{array}{l}\text { Existe un } 32 \% \text { de rezago en el tema de números enteros y } \\
\text { sus operaciones, resta con punto decimal. }\end{array}$ \\
\hline 6. & $(3-8)+[5-(-2)]$ & $\begin{array}{l}\text { Existe un } 30 \% \text { de rezago en el tema de números enteros y } \\
\text { sus operaciones, operaciones con signos de agrupación. }\end{array}$ \\
\hline 7. & $5-[6-2-(1-8)-3+6]+5$ & $\begin{array}{l}\text { Existe un } 46 \% \text { de rezago en el tema de números enteros y } \\
\text { sus operaciones, operaciones con signos de agrupación. }\end{array}$ \\
\hline 8. & {$\left[(17-15)^{3}+(7-12)^{2}\right]:[(6-7) \cdot(12-23)]$} & $\begin{array}{l}\text { Existe un } 65 \% \text { de rezago en el tema de números enteros y } \\
\text { sus operaciones, operaciones con signos de agrupación y } \\
\text { potenciación y radicación. }\end{array}$ \\
\hline 9. & $(7-2+4)-(2-5)$ & $\begin{array}{l}\text { Existe un } 43 \% \text { de rezago en el tema de números enteros y } \\
\text { sus operaciones, operaciones con signos de agrupación. }\end{array}$ \\
\hline 10. & $6+\{4-(17-(4 \cdot 4)]+3\}-5$ & $\begin{array}{l}\text { Existe un } 43 \% \text { de rezago en el tema de números enteros y } \\
\text { sus operaciones, operaciones con signos de agrupación. }\end{array}$ \\
\hline 11. & $\begin{array}{l}\text { Teresa ve en el pizarrón la siguiente fracción: } 10.17 \text {, } \\
\text { ¿Cuál de las siguientes opciones muestra correctamente } \\
\text { en escritura decimal esa fracción? } \\
\text { A) Un entero, siete décimos. } \\
\text { B) Diez y siete milésimos. } \\
\text { C) Diez y siete centésimos. } \\
\text { D) Un entero, siete centésimos. }\end{array}$ & $\begin{array}{l}\text { Existe un } 32 \% \text { de rezago en el tema de números enteros } \\
\text { y sus operaciones, problema que involucra los números } \\
\text { decimales. }\end{array}$ \\
\hline
\end{tabular}

12. En una competencia de canotaje compiten 4 países, cada país cuenta con 4 canoas y cada canoa cuenta con 4 participantes. ¿Cuántos participantes hay en total dentro de Existe un $0 \%$ de rezago en el tema de números enteros y la competencia? sus operaciones, problema que involucra potenciación y A) 12 B) 20 C) 32 D) 64

13 El área de un muro rectangular es de $3,230 \mathrm{~m}^{2}$, si la base mide $312 \mathrm{~m}$, ¿cuántos metros mide la altura del muro? A) 7.113 B) 3.128 C) 3.132 D) 9.571

radicación.

Existe un $57 \%$ de rezago en el tema de números enteros y sus operaciones, problema que involucra división y despeje, la interpretación de resultado (la altura del muro es $10.352 \mathrm{~m})$.

14. Juan va a colocar losetas de $0.30 \mathrm{~m} \times 0.30 \mathrm{~m}$ en el piso de una de las habitaciones de su casa que mide $15 \mathrm{~m}^{2}$ : ¿cuál es el número aproximado de losetas que empleará? A) 14.97 B) 15.48 C) 43 D) 86

Existe un $87 \%$ de rezago en el tema de números enteros y sus operaciones, problema que involucra números decimales, multiplicación con números decimales, división con números decimales, interpretar la solución (el número de losetas es 166.66 , se interpreta 167 losetas y no hay un número aproximado).

Nota: Elaboración propia. 


\section{Tabla 4. Secuencia didáctica (Díaz-Barriga, 1984)}

\begin{tabular}{ll}
\hline Asignatura: & Matemáticas IV \\
\hline Autores: & $\begin{array}{l}\text { María del Socorro Rivera Casales, Ricardo Jacob Mendoza Rivera, Andrea Cárdenas } \\
\text { Mendoza }\end{array}$ \\
\hline Número y nombre de la unidad: & $\begin{array}{l}\text { Unidad 1. Los números reales para contar, comparar y medir } \\
\text { Número de horas: } 25\end{array}$ \\
\hline Nombre de la actividad: & "EL ÁBACO NEPOHUALTZINTZIN, RESCATANDO LA ETNOMATEMÁTICA EN CÁL- \\
CULOS ARITMÉTICOS"
\end{tabular}

Tema y problema o situación que
se aborda:

El tema es la innovación del material didáctico el ábaco Nepohualtzintzin, mediante una propuesta de secuencia didáctica para fortalecer las habilidades aritméticas, relacionándola con temas del curso, para lograr un aprendizaje significativo en alumnos de IV de la ENP \#2; así se nivela a los alumnos en operaciones aritméticas. Problema: Rezago existente de los alumnos en operaciones aritméticas con un aprendizaje significativo. Fortalecer las habilidades aritméticas, innovando con el ábaco Nepohualtzintzin, definido como material didáctico para lograr un aprendizaje significativo, al inicio del curso a los alumnos de cuarto año del plantel \#2, con el grupo 417, dentro de sus horarios de clase, en el ciclo escolar 2019-2020.

\section{Objetivo general}

El alumno elaborará representaciones matemáticas de una situación real, mediando el Objetivos: ábaco Nepohualtzintzin, realizando procesos de abstracción y generalización, que le permitan valorar el potencial de la etnomatemática en su formación como ciudadano crítico y consciente de su entorno, y en su preparación académica para fortalecer los cálculos aritméticos y avanzar con conocimientos firmes en los temas posteriores.

1.1 Conjunto de los números reales y sus subconjuntos.

1.2 Problemas que involucran razones y proporciones.

1.3 Leyes de los exponentes.

1.4 Representación de información numérica en forma gráfica (en la recta numérica, en el plano cartesiano, o usando sectores circulares, barras, histogramas, etc.), realizada manualmente y con apoyo de software matemático.

1.5 Modelación de situaciones que requieren el uso de números reales (naturales, enteros, racionales e irracionales).

Contenidos que se abordan:

1.6 Aplicación de la jerarquía y las propiedades de los diferentes subconjuntos de los números reales en la resolución de operaciones que incluyen potenciación/radicación, multiplicación/división y adición/sustracción, para obtener la expresión más simple.

1.7 Representación de los números racionales e irracionales mediante su expansión decimal.

1.8 Valoración de la importancia de los números para contar, medir y comparar.

1.9 Valoración de la importancia de justificar un procedimiento utilizando la aplicación de las propiedades estudiadas, para desarrollar su razonamiento lógico.

1.10 Concientización de que representar y analizar información numérica permite fundamentar una opinión y establecer una postura personal.

25 sesiones de enseñanza, con una duración de una hora, bajo la guía de los ejercicios del libro CONAMAT (2015). Matemáticas Simplificadas. Pearson.

Duración (número de sesiones):

Nota: Elaboración propia con datos de Plan de Estudios ENP.

El proyecto de investigación basado en el análisis del cuestionario diagnóstico antes del curso confirma que es necesario dar una solución al rezago existente en operaciones aritméticas con aprendizaje significativo (a largo plazo). La propuesta es elaborar 25 sesiones de aprendizaje, mediadas por la propuesta de secuencia didáctica. Las sesiones de enseñanza llevan a la elaboración de un manual de trabajo con los temas de aritmética que obtuvieron más errores, empleando el ábaco Nepohualtzintzin para una mejor comprensión en la enseñanza-aprendizaje (Rivera, 2012b). A continuación, se presenta el análisis de la secuencia didáctica planteada en el desarrollo de la investigación (Tabla 5). 


\section{Tabla 5. Análisis general de la secuencia didáctica}

\begin{tabular}{lll}
\hline Resultados del cuestionario & Análisis de la falta de interés & Análisis del rezago \\
\hline El promedio para resolver el cuestiona- & El 23\% (9 alumnos) de los estudiantes & Se concluye en promedio que el $36 \%$ \\
rio fue de 39 minutos (resolver 14 reac- & muestra falta de interés al resolver los & (14 alumnos) de los estudiantes tiene un \\
tivos); el $22 \%$ ( 8 alumnos) no tiene un reactivos (solo respondieron en pro- & rezago en operaciones aritméticas con \\
aprendizaje memorístico receptivo (no medio 4 reactivos); el $1 \%$ (5 alumnos) & un aprendizaje memorístico receptivo \\
saben resolver los ejercicios con opera- resolvió el cuestionario completo. Se & antes del curso, y un $92 \%$ (34 alumnos) \\
ciones); el $78 \%$ ( 29 alumnos) tiene un concluye que el $99 \%$ (32 alumnos) tiene & tiene un rezago en la enseñanza del \\
rezago en el aprendizaje memorístico rezago en el interés para realizar opera- & aprendizaje memorístico por descubri- \\
por descubrimiento propio (solo meca- ciones aritméticas. & miento autónomo (no llegan a un apren- \\
nizan la solución). Se concluye que el & dizaje a largo plazo). \\
$100 \%$ (los 37 alumnos) tiene un rezago & \\
en aprendizaje memorístico por des- & \\
cubrimiento autónomo (no llegan a un & \\
aprendizaje a largo plazo). &
\end{tabular}

Nota: Elaboración propia.

Un resultado importante del análisis arroja, en promedio, que el 97\% de los alumnos (35) tiene un rezago al realizar el cuestionario diagnóstico antes del curso, por las causas señaladas en la Tabla 4.

Sobre el aprendizaje del alumno se presenta en detalle el análisis comparativo de tipos de aprendizaje con respuestas al reactivo 7 del cuestionario diagnóstico.

La Figura 3 presenta la solución al reactivo 7 con un aprendizaje memorístico receptivo por algoritmos matemáticos. Este tipo de aprendizaje es señalado por Díaz-Barriga (2010).

\section{Figura 3. Respuesta de un estudiante por aprendizaje memorístico receptivo por algoritmos matemáticos}

$$
\text { 7. } 5-[6-2-(1-8)-3+6]+5=-4
$$

Justificación del alumno:

$$
\begin{gathered}
5-[6-2-(1-8)-3+6]+5= \\
5-[6-2-(-7)-3+6]+5 \\
{[6-2+7-3+6]} \\
5-[+14]+5 \\
+5-14+5 \\
-9-4
\end{gathered}
$$

Nota. Se transcribe la justificación para efectos editoriales. Fuente: Evidencia fotográfica del proyecto. 
La solución de la Figura 4 corresponde a las respuestas de una alumna después de la primera sesión de enseñanza (uso básico del ábaco), con un tipo de aprendizaje significativo por descubrimiento guiado, con una instrucción bien diseñada, como lo señala Díaz-Barriga (2010).

$$
5-[6-2-(1-8)-3+6]+5=
$$

Paso 1. Se representa la operación dentro del paréntesis: $6,-2,-(-7),-3 y+6$, se efectúa y el resultado es -7 . Para representarlo en el ábaco es necesario bajar una ficha de valor 5 y sumarle dos de valor uno.

\section{Figura 4. Señala en el ábaco Nepohualtzintzin el paso 1}

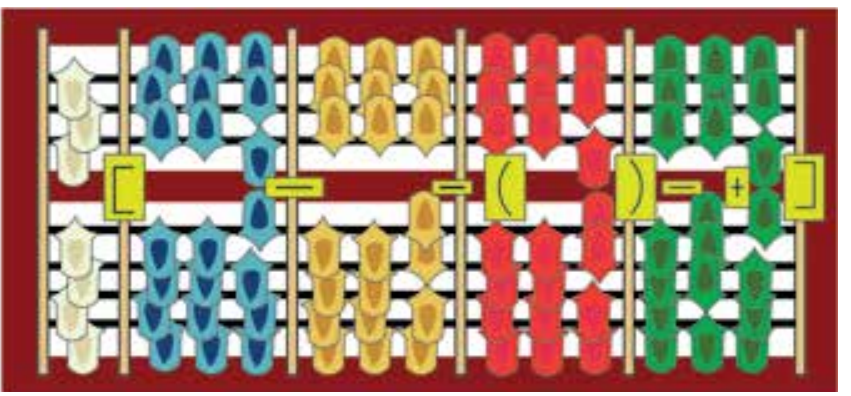

Fuente: Elaboración propia basada en información fotográfica del proyecto

Paso 2. Se elimina el paréntesis gracias a la ley de los signos $(-)+(-)=(+)$ y así queda como resultado un 7 positivo, que también se representa bajando una ficha de valor 5 y sumando dos de valor uno; representamos el 6 , el $-2,+7$ y se realiza la operación $-3+6=3$.

\section{Figura 5. Señala en el ábaco Nepohualtzintzin el paso 2}

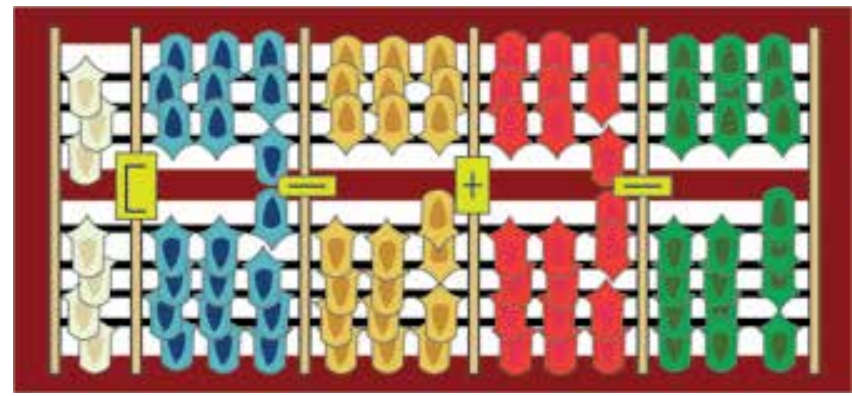

Fuente: Elaboración propia basada en información fotográfica del proyecto
Paso 3. Se sustituyen los valores para poder obtener 14: bajando una ficha con valor de $10 \mathrm{y}$ subiendo cuatro con valor de uno. Se simplifica la operación a 10 - 14 y da como resultado - 4. Esto se representa subiendo 4 fichas con valor de 1 y se indica el signo, como lo muestra la Figura 6.

\section{Figura 6. Señala en el ábaco Nepohualtzintzin el paso 3}

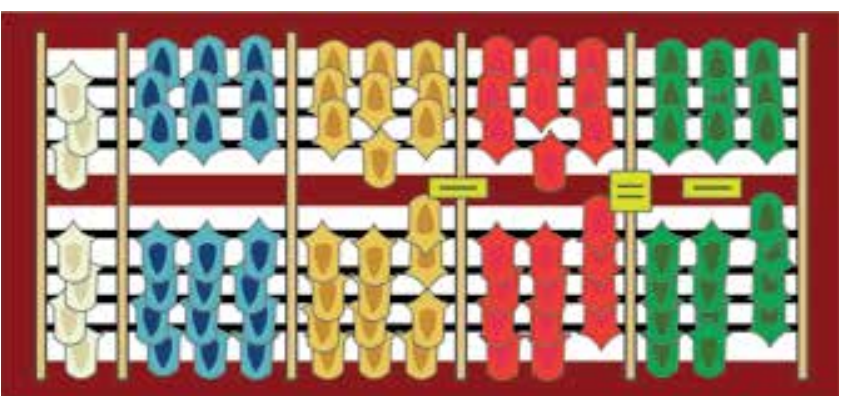

Fuente: Elaboración propia basada en información fotográfica del proyecto.

Se concluye del análisis de la resolución del reactivo 7, por tipo de aprendizaje memorístico-receptivo (algoritmos matemáticos o resolución por operaciones aritméticas) y por tipo de aprendizaje significativo por descubrimiento-guiado, un cambio muy significativo al no presentar errores y llegar al resultado correcto. $\mathrm{Al}$ entrevistar a la alumna, su respuesta fue que prestó más atención en cada paso para llegar a la respuesta correcta, le agradó hacer el reactivo de una manera diferente a lo que hace comúnmente en clase y mencionó que la sacó de su zona de confort.

Los investigadores en matemática educativa (Rivera, 2012a) tienen amplias y profundas investigaciones en este campo; es importante reconocer los esfuerzos de las comunidades académicas nacionales e internacionales por replantear el paradigma de la educación y dar soluciones distintas a los problemas planteados en las investigaciones.

Es necesario en la enseñanza-aprendizaje bien organizada que los docentes e investigadores busquen estrategias diferentes, secuencias fundamentadas, materiales didácticos (digitales o manuales), situaciones didácticas novedosas, para mejorar el 
proceso de enseñanza-aprendizaje, motivando a los alumnos a realizar actividades diferentes a las acostumbradas. Así se lograría reducir el rezago y se mejoraría el razonamiento lógico-matemático que apoya el desarrollo de capacidades cerebrales a través de la conexión mente-cuerpo por medio del movimiento.

Se termina este artículo con la construcción de un triángulo de conocimiento (Figura 5) que es de gran do por el ábaco Nepohualtzintzin.

\section{Figura 5. Construcción del triángulo de conoci- miento}

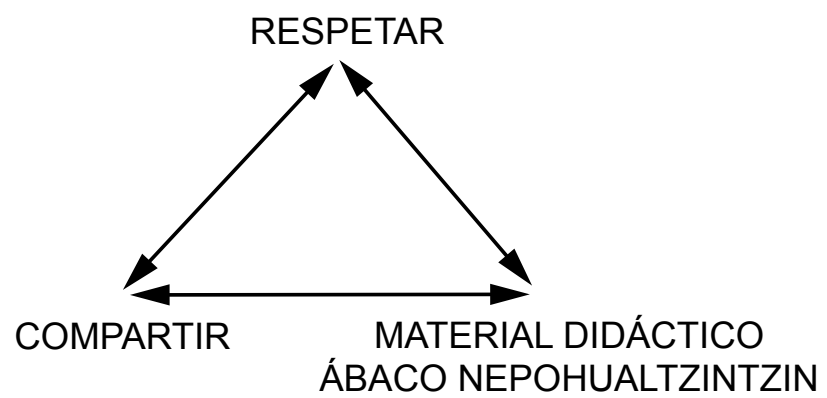

Nota: Elaboración propia

La construcción es una secuencia de palabras vinculadas entre sí que constituyen una unidad pedagógica llena de saberes. El triángulo se formó con la figura geométrica más usada en las culturas mesoamericanas, las majestuosas pirámides. Cada vértice tiene una razón importante en el trabajo continuo del aula real, a saber:

1) RESPETO. Primero que nada, el respeto como ser humano, con cualidades y errores, al trabajo en escuelas públicas o privadas, a los alumnos, a las autoridades, a la institución, a los prehispánicos, a los dioses, a la naturaleza.

2) COMPARTIR. Es repartir el conocimiento en cada especialidad que tenemos, dividir la experiencia y, lo más importante, distribuir la enseñanza-aprendizaje.

3) MATERIAL DIDÁCTICO ÁBACO NEPOHUALTZINTZIN. Ayuda a presentar y desarrollar los contenidos para que los alumnos trabajen en la construcción del conocimiento, llegando en consecuencia a la obtención de aprendizajes significativos.

\section{Agradecimientos}

A las personas involucradas en la investigación: alumnos de la ENP, UNAM, COLPOS.

\section{Referencias}

Anijovich, R. (2010). La evaluación significativa. Paidós.

Ausubel, D. (1976). Psicología educativa. Trillas.

Brousseau, G. (2007). Iniciación al estudio de la teoría de las situaciones didácticas. Libros Zorzal.

Coleman, J., Campbell, E., Hobson, C., McPartland, J., Mood, A., Weinfeld, F., \& Cork, R. (1966). Equality of Educational Opportunity. U.S. Government Printing Office.

D’Ambrosio, U. (2008). Etnomatemática. Eslabón entre las tradiciones y la modernidad. Limusa.

Díaz-Barriga, F. (1984). Didáctica y Currículum. Articulaciones en los programas de estudios. Nuevomar.

Díaz-Barriga, F. (2004). Enseñanza situada. McGraw Hill.

Díaz-Barriga, F. (2010). Estrategias docentes para un aprendizaje significativo. McGraw-Hill.

Cárdenas, A. (2016). Propuesta de plan y programas de doctorado en ciencias de la administración modalidad no escolarizada para su inscripción al RVOE. [Tesis doctoral, Colegio de Estudios de Posgrado de la Ciudad de México]. Cuernavaca, Morelos.

Eisner, E. (1998). El ojo ilustrado. Indagación cualitativa y mejora de la práctica educativa. Paidós.

Escuela Nacional Preparatoria (1996). Plan de estudios 1996. Clave 1403. Universidad Nacional Autónoma de México. Escuela Nacional Preparatoria. Aprobado por el H. Consejo Técnico 2016. 
https://r.issu.edu.do/l?l=10205gJL

Escuela Nacional Preparatoria (1996). Plan de estudios 1996. Clave 1400. Universidad Nacional Autónoma de México. Escuela Nacional Preparatoria. Aprobado por el H. Consejo Técnico 2016.

https://r.issu.edu.do/l?l=10206mnv

Flores S. (2009). Manual didáctico del nepohualtzitzin para el desarrollo de las competencias matemáticas. CGEIB-SEP.

Jaramillo, D., Torres, B., \& Villamil, M. (2006). Interacciones en clase de matemáticas: una mirada desde la etnomatemática. Foro Educativo Nacional 2006.

León-Portilla, M. (2011). Cantares mexicanos: Vol. I. Universidad Nacional Autónoma de México.

López-Suárez, A., Morales-Hernández, I., \& Silva-Beltrán, E. (2005). El sostenimiento de la educación en México. Papeles de Población, 11(44), 239-254.

López, S. (2008). Ábaco. Revista Unión, 14, 153-156.

Novak, J. D., \& Gowin, D. B. (1988). Aprendiendo a aprender. Martínez Roca.
Ojeda, A. M. (2006). Estrategia para un perfil nuevo de docencia: Un ensayo en la enseñanza de estocásticos. En E. Filloy (Ed.), Matemática Educativa, 30 años. Una mirada fugaz, una mirada externa y comprensiva, una mirada actual. Santillana.

Real Academia Española (2019). Diccionario de la Lengua Española. https://dle.rae.es/\%C3\%A1baco

Rivera, S. (2012a). Comprensión de ideas fundamentales de estocásticos en el Bachillerato. Editorial Académica Española.

Rivera, S. (2012b). Una reflexión en la enseñanza de las matemáticas. Editorial Académica Española.

Soto, C. (2014). Manual de Uso y Ejercicios Nepohualtzintzin (ábaco azteca).

https://r.issu.edu.do/l?l=10207Qn7

Vázquez, M. (2010). Materiales didácticos para matemáticas. Eroski Consumer. https://r.issu.edu.do/l?l=10208KRv

\section{CÓMO CITAR:}

Rivera-Casales, M. S., Mendoza Rivera, R. J., \& Cárdenas Mendoza, A. (2021). Propuesta de secuencia didáctica utilizando el ábaco Nepohualtzinzin para los cálculos aritméticos. RECIE. Revista Caribeña de Investigación Educativa, 5(2), 7-22. https://doi.org/10.32541/recie.2021.v5i2.pp7-22 